\title{
Light and Electron Microscopic Studies of Kidneys in Toxemia of Pregnancy by Means of Renal Biopsy
}

\author{
By \\ Masayoshi Yamazaki
From the Department of Gynecology, Director; Prof. K. Kushima and the First Department of Pathology, Director: Prof. N. Suwa
Tohoku University School of Medicine, Sendai

(Received for publication, August 24, 1965)

\begin{abstract}
Light and electron microscopical examinations were performed on kidney specimens obtained from 52 cases with toxemia of pregnancy by means of needle biopsy. The main renal injuries were found in glomeruli. Glomerular lesions were initiated by precipitation of plasmal elements in lamina rara interna and followed by invasion of mesangial cells which formed membraneous structures resembling lamina densa. The structures were recognized in histological sections as the characteristic reduplication of capillary basement membrane.

Distinct correlation was confirmed between the intensity of glomerular involvement and proteinuria. In cases with conspicuous glomerular lesions immediately after delivery, protracted proteinuria over months was generally observed. Glomerular lesions had, however, no bearing on the development of persisting hypertension after toxemia of pregnancy. The development of chronic glomerulonephritis on the basis of toxemia of pregnancy was not confirmed.

In cases with prolonged hypertension after delivery, the second biopsies revealed more or less pronounced fibroelastic hyperplasia of arteriolar intima. The lesions were regarded to belong to results, not to causes, of hypertension.
\end{abstract}

Toxemia of pregnancy is clinically characterized by the triad, edema, proteinuria and hypertension. Because all the symptoms are generally closely associated with renal diseases, it has been expected that anatomical studies of kidneys in toxemia of pregnancy would contribute to elucidate the pathogenesis of the syndrome. The kidneys in toxemia of pregnancy were first studied by Leyden, ${ }^{10}$ who regarded the renal changes to be degenerative in nature and different from those in Bright's diseases. His conclusion led to further investigations on the problem, whether renal changes in the syndrome could histologically be characterized as an independent disease entity or not. Leyden pointed out swelling and ischemia of renal glomeruli in toxemia of pregnancy. Löhlein ${ }^{11}$ regarded that glomeruli were the principal site of renal injuries in this disease and confirmed proliferation of endothelial cells and thickening of glomerular capillary walls. He

山崎正義 
believed that the histological findings could be distinguished from those in glomerulonephritis, although the discrimination was impossible in cases of slight glomerular involvement. His opinion was accepted by $\mathrm{Fahr}^{5}$ who described distinctly swollen glomeruli which sometimes completely filled Bowman's capsules. According to Fahr, ${ }^{5}$ glomerular changes in toxemia of pregnancy were characterized by swelling and thickening of capillary walls of glomeruli. When swelling of capillary walls was conspicuous, capillary loops were voluminous and capillary lumina were so narrowed as to expel erythrocytes. He regarded the above glomerular change to be degenerative in character, devoid of endothelial proliferation, and to be different from that of glomerulonephritis.

Bell ${ }^{3}$ attributed swelling of capillary loops almost exclusively to thickening of capillary basement membrane in his histological study on 20 autopsy cases. Swollen capillary basement membrane was regarded to cause stenosis of capillaries. He described in his first report that only minimum changes were observed in capillary endothelial cells, but he later recognized endothelial proliferation. Baird and Dann ${ }^{2}$ observed uneven thickening of capillary basement membrane. He described further that capillary lumina were obliterated by swollen and bulky endothelial cells with highly rarified and almost foamy cytoplasm. He stated that the lesion was diffuse in character and approximately uniformly distributed throughout the entire kidney. The majority of investigators observed thickened basement membrane of glomerular capillaries, not only in eclamptic patients, but also in those in pre-eclamptic conditions. According to Allen, ${ }^{1}$ glomerular lesions in these conditions belonged to acute or chronic membraneous glomerulonephritis and it was practically impossible to discriminate them from chronic membraneous glomerulonephritis of other origins in its initial stage. Rinehart et al. ${ }^{13}$ also recognized the resemblance of eclamptic glomerular lesions to membraneous glomerulonephritis. However, Kark et al. ${ }^{9}$ did not support in his light microscopic investigation the opinion of Allen. Farquhar ${ }^{6}$ did not acknowledge the resemblance of glomerular lesions in pre-eclamptic state to those of lipoid nephrosis or membraneous glomerulonephritis on the basis of her electron microscopic observations. According to Govan, Canny and McManus, ${ }^{12}$ glomerular changes did not consist in thickening of basement membrane, but in thickening of mesangial tissue and local proliferation of mesangial cells. McManus, ${ }^{12}$ who found no evidence of thickening of glomerular basement membrane, described fine reticulation and vacuolization of endothelial cells, which he ascribed to edema of glomerular tufts. Sharma ${ }^{14}$ also described edema of glomeruli.

Electron-microscopically, Farquhar ${ }^{6}$ found distinct swelling of endothelial cytoplasm and subendothelial deposit of fibrinoid substance. Ishikawa et al. ${ }^{8}$ found swelling of epithelial cells but reported that there was no change in the basement membrane of glomerular capillaries. Spargo et al. ${ }^{15}$ reported that increase of endothelial cytoplasm was characteristic to toxemia of pregnanacy and called 
the finding glomerular capillary endotheliosis.

Pathological findings of renal tubules, interstitial tissues and vascular system are relatively unimportant and secondary in character. Cloudy swelling and hydropic degeneration of epithelial cells with occasional protein-like intratubular precipitates, fat or hyaline droplets in epithelial cells have often been reported. Severe degenerative changes including tubular necrosis was only rarely observed. Russel described interstitial edema in kidneys of toxemia of pregnancy. Govan ${ }^{7}$ reported enlargement of juxtaglomerular apparatus. McManus ${ }^{12}$ observed increased granularity of the cell group of the above structure. Localized thickening of afferent arteries was noticed by Fahr, ${ }^{5}$ and Govan ${ }^{7}$ described irregular lumina of afferent arteries due to eccentric swelling of their walls. Swelling of the wall of afferent arteries was also reported by Way. ${ }^{16}$

The majority of hitherto reported investigations demonstrated glomeruli as the most important site of renal lesions in toxemia of pregnancy. Perfect agreement is, however, not reached on the nature of glomerular changes. Furthermore, although these investigations revealed distinct involvement of the kidney in toxemia of pregnancy, there were few studies on the relations between morphological changes and clinical symptoms. The purpose of the present study is to examine morphological changes of the kidney in correlation to clinical symptoms.

\section{MATERIALS AND METHODS}

Pregnant women in gynecological clinic of Tohoku University who had at least one of the following symptoms were investigated.

1) The maximum blood pressure over $140 \mathrm{mmHg}$, or the minimum blood pressure over $100 \mathrm{mmHg}$.

2) Proteinuria detected by sulfosalicylic acid test.

3) Subcutaneous edema.

Fifty-two biopsy specimens were obtained from the kidneys of these patients. The obtained renal tissues were $1-3 \mathrm{~cm}$ long. They were washed in physiological saline for a short time, and small tissue pieces for electron microscopsy were excised from both ends. The specimens were fixed for 2 hours at $0^{\circ} \mathrm{C}$ in $2 \%$ osmic acid solution of $\mathrm{pH} 7.4$ buffered with acetate-veronal. Remaining tissue pieces were placed in acidified Zenker-formol solution and submitted to light microscopic studies. Paraffin sections of 3 micron thickness were prepared and stained with hematoxylin-eosin, PAS, elastica-Goldner's and Azan-Mallory's. The specimens for electron microscopy were dehydrated by alcohol and embedded in buthylmethacrylate mixed with $20 \%$ methylmethacrylate. They were cut at 20-40 millimicrons and examined under Hitachi HU-10 type electron microscope. Most specimens were studied without stain but a small number of tissues was examined after lead or uranyl stain. 
The investigated patients were re-examined 1-3 months after delivery for blood pressure, urine (protein, sugar, urobilinogen) and other clinical symptoms. When some abnormalities were observed, the patients were further exmained 11.5 years later. Out of examined 52 cases, 8 had only edema, 14 proteinuria alone, 11 hypertension and 19 had both proteinuria and hypertension at the time of biopsy.

\section{RESULTS}

Histologically confirmable renal changes were observed in 47 out of 52 cases. Glomerulus was the main site of renal injury and glomerular lesions were confirmed in 31 cases. Glomerular involvement was almost uniform and diffuse in character. Glomeruli were swollen and enlarged in the majority of cases. This was mainly due to thickening of mesangial tissue and capillary basement membrane. In light microscopical examinations, the lesion appeared to be edema of mesangial tissue which extended very often to capillary basement membrane. In the majority of the cases, thickened mesangial tissue contained protein-like precipitates which were demonstrated as faintly yellow or red amorphous substances by Azan staining. In some cases the precipitates were of distinctly fibrinoid character, and in extreme cases capillary basement membranes were diffusely thickened on account of subendothelial fibrinoid precipitates and exhibited wire-loop appearance.

One of the most important glomerular changes was the "splitting" of capillary basement membrane. The membrane was demonstrated in reduplicated lines in histological sections. The nature of the finding is to be revealed by electron microscopy.

Electron-microscopically, endothelial cells were partially swollen and vacuolated. They often narrowed the capillary lumina. In advanced lesions swollen and vacuolated endothelial cells could completely occlude capillary lumina. Endothelial cells were reduced in their electron density, and liquid element was probably increased in their cytoplasm. The patients who had proteinuria at biopsy generally exhibited prominent changes of lamina rara interna of glomerular capillaries. The layer was widened apparently on account of edema with occasional coarse and similar granules, which corresponded to the precipitation of plasmal elements in the findings of light microscopic examinations. When the lesion was fully developed, the layer was densely filled by granular substances. Even in cases with dense precipitations in lamina rara interna, the layer was clearly distinguished from homogeneous lamina densa which did not exhibit remarkable changes. The precipitations were only observed in lamina rara interna and not in lamina rara externa.

Apparently subsequent to the above lesions, mesangial cells began to proliferate and to invade lamina rara interna, which was indicated by the 
appearance of membraneous substances and intracellular structures characteristic to mesangial cells. In initial stages, extensions of mesangial cells from mesangial side were observed into lamina rara interna, which was still interspersed with granular substances. In advanced lesions, mesangial cell extensions surrounded the entire circumference of capillaries, provided with nuclei of mesangial type and newly formed membraneous structures. The latter structures were of the same electron density with lamina densa. Light microscopically demonstrated double lines or "splitting" of capillary basement membrane is evidently due to the formation of membraneous structure by invading mesangial cells.

In spite of the conspicuous changes of lamina rara interna and mesangial cells, there was surprisingly little lesion in lamina densa itself. In only one of the 52 examined cases, unevenness of the layer due to its irregular thickening could be demonstrated. It is doubtful, whether the finding is to be attributed to toxemia of pregnancy or not. Glomerular epithelial cells were swollen, with abundant granules and vacuoles. Their foot processes were fused together and flattened.

Histologically, epithelial cells of proximal convolution were in the majority of cases swollen on account of albuminous degeneration or of hyaline droplets. However, there was neither histological nor electron microscopical lesions specific to toxemia of pregnancy. The most noteworthy finding was cortical necrosis observed in two cases.

Slight hyaline thickening and fibroelastic intimal hyperplasia were found in small arteries and arterioles in some of the cases, but fibrinoid deposit was not demonstrated.

In 3 patients out of the total examined group one or more of the clinical symptoms persisted over years after delivery. Their kidneys were examined by means of the second biopsy. In the first case, renal biopsy immediately after delivery revealed distinct glomerular involvement in the form of "splitting" of capillary basement membrane. Vascular changes were not observed at that time. The patient developed prolonged and persistent hypertension without albuminuria. At the second biopsy, glomerular lesions were already subsided, and only slight hyaline scar of mesangial tissues indicated previous glomerular involvement. There was no progressive glomerular lesion in the second biopsy specimen. On the contrary, fibroelastic thickening of arteriolar intima was distinctly developed. In the other two cases, cortical necrosis was found in the first biopsy specimens, together with distinct glomerular changes, consisting mainly in edematous swelling of mesangial tissues. In one of the cases, hypertension after the delivery persisted over years, and fibroelastic thickening of the arteriolar intima was observed at the second biopsy. Glomerular lesions were, however, not conspicuous and only slight hyaline thickening of mesangial tissues and double structure of capillary basement membrane were still confirmable. In the other case which had only proteinuria without hypertension, no remark- 
able vascular change was observed at the second biopsy. Glomerular lesions were also inconspicuous in spite of persisting proteinuria, and mesangial tissues were in the state of scar.

\section{COMMENTS}

Since Bell ${ }^{3}$ reported that the major renal lesion in toxemia of pregnancy consisted in edematous thickening of capillary basement membrane, his opinion has been supported by a number of following investigators. As Bell did not recognize mesangial tissue as a constituent structure of glomerulus, the described glomerular changes must have included not only the lesions of capillary basement membrane in the strict sense of the word, but also edematous swelling and slight and occasional proliferation of mesangial tissues. He also confirmed reduplicated structure of capillary basement membrane and precipitation of yellow and red substances in Mallory stained sections, and ascribed the finding to edema of capillary basement membrane. In the present study, in which light microscopical findings were complemented by electron microscopic observations, the development of glomerular lesions could be followed up with greater accuracy.

Glomerular changes in toxemia of pregnancy appeared to be initiated electron-microscopically by the precipitation of granular substances in lamina rara interna. This was probably due to enhanced endothelial permeability and to infiltration and subsequent condensation of plasmal elements. The lesion corresponded to subendothelial precipitation of yellow or red amorphous substances in Mallory stained sections. Up to this phase, glomerular lesions could be regarded to be edema of capillary basement membrane and probably likewise of mesangial tissues in the proper sense of the word. However, this was almost immediately followed by invasion of mesangial cells into lamina rara interna. The most conspicuous finding of this phase was the formation of membraneous structures of the same electron density to that of lamina densa. Some investigators assert, that the finding is due to splitting of lamina densa as a result of mesangial invasion. The present investigation did not support the view, for mesangial invasion in lamina rara interna without membraneous structures could be confirmed in the initial stage of the process. Lamina densa was not only completely free from any pathological change in this phase, but also quite inactive in the entire development of glomerular lesions in toxemia of pregnancy.

The result of the present electron microscopic study in relation to histological findings revealed that at least two different processes were comprised in lightmicroscopically observed "edema" of capillary basement membrane. One of them was the precipitation of plasmal elements in lamina rara interna and was regarded to be proper edema. The process is light-microscopically recognized as subendothelial precipitation of yellow or red substances in Mallory stained sections. The other is the so-called "splitting" or reduplciation of capillary basement 
membrane. Because of the difficulty to demonstrate intracellular structures in light microscopical examinations, this finding has likewise been assumed to be one of the expressions of edema in capillary basement membrane. Electron microscopical exmainations revealed, however, that the process was in reality induced by mesangial invasion and new formation of membraneous structure resembling lamina densa. This process could not consequently be regarded as one of the expressions of edema, but as slight proliferation of mesangial cells. In this respect, renal lesions in toxemia of pregnancy were to some part related to diffuse proliferative glomerulonephritis, although the intensity of proliferation was much lower and attained the grade of histologically demonstrable proliferative glomerulonephiritis in only a single case.

The intensity of histologically confirmable glomerular lesions was closely related to proteinuria. Distinct correlation of the histological findings of renal biopsy specimens immediately after delivery was demonstrable to proteinuria just after delivery and to protracted proteinuria over months after delivery. Remnant proteinuria over months was not, however, due to progressive glomerular lesions, and the author could not detect a single case at the second biopsy, in which chronic glomerulonephritis developed on the basis of toxemia of pregnancy. On the contrary, there was no correlation whatever between glomerular lesions and hypertension. Durable and persistent hypertension could develop even in cases without or with only slight glomerular involvement. There was also no correlation between blood pressure level and glomerular changes at delivery. In cases of prolonged hypertension the second biopsies revealed more or less conspicuous vascular lesions mainly in the form of intimal fibroelastosis of arterioles, but no progressive glomerular lesion. The former lesions were evidently one of the results, not the causes, of the hypertension. Farquhar ${ }^{6}$ reported undula-

Albuminuria and Glomerular Lesion of Delivery

\begin{tabular}{|c|c|c|c|c|c|}
\hline $\begin{array}{c}\text { Number of } \\
\text { Coses }\end{array}$ & II & 4 & 11 & 5 & 31 \\
\hline H & & & 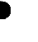 & 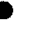 & 2 \\
\hline$H$ & & & & & 7 \\
\hline+ & & & & & 6 \\
\hline \pm & & 10 & 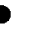 & & 4 \\
\hline- & & & 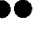 & & 12 \\
\hline Les & - & \pm & + & $H$ & $\begin{array}{c}\text { Number } \\
\text { ot } \\
\text { Coses }\end{array}$ \\
\hline
\end{tabular}

Fig. 1. Distinct positive correlation is indicated between glomerular lesions and albuminuria immediately after delivery. 


\begin{tabular}{|c|c|c|c|c|c|}
\hline $\begin{array}{c}\text { Number of } \\
\text { Coses }\end{array}$ & 6 & 4 & 8 & 5 & 23 \\
\hline \# & & & & & 0 \\
\hline$H$ & & & & 0 & 1 \\
\hline+ & & 0 & $\infty$ & - & 4 \\
\hline \pm & & 0 & 0 & $\infty$ & 6 \\
\hline- & t) & 00 & & & 12 \\
\hline Lesion & - & \pm & $t$ & $H$ & $\begin{array}{c}\text { Number } \\
\text { of } \\
\text { Cases }\end{array}$ \\
\hline
\end{tabular}

Fig. 2. Correlation between glomerular lesions at delivery and albuminuria observed a month after delivery. Note still evident correlation. Persistent albuminuria over months can be predicted on the basis of histological findings immediately after delivery.

Hypertension and Glomorular Lesion of Delivery

\begin{tabular}{|c|c|c|c|c|c|}
\hline $\begin{array}{c}\text { Number } \\
\text { of } \\
\text { Coses }\end{array}$ & 11 & 4 & 10 & 6 & 31 \\
\hline+ & $\bullet 8^{\circ}$ & 1 & $88^{\circ}$ & $\bullet$ & 13 \\
\hline- & 88 & $\bullet \bullet \bullet$ & $88^{\circ}$ & $8^{\circ}$ & 18 \\
\hline $\begin{array}{l}\text { Hyper- } \\
\text { rension } \\
\text { Gesion }\end{array}$ & - & \pm & + & $H$ & $\begin{array}{c}\text { Number } \\
\text { of } \\
\text { cases }\end{array}$ \\
\hline
\end{tabular}

Fig. 3. In contrast to albuminuria, there is no relation between blood pressure level and glomerular lesions.

\begin{tabular}{|c|c|c|c|c|c|}
\hline $\begin{array}{c}\text { Number } \\
\text { of } \\
\text { Coses }\end{array}$ & 6 & 4 & 8 & 5 & 23 \\
\hline+ & 0 & 0 & 0 & 0 & 4 \\
\hline- & 88 & 000 & 889 & 800 & 19 \\
\hline $\begin{array}{c}\text { Hyper-_ } \\
\text { tonsion GI. }\end{array}$ & - & \pm & + & $H$ & $\begin{array}{c}\text { Mumber } \\
\text { of } \\
\text { Coses }\end{array}$ \\
\hline
\end{tabular}

Fig. 4. Persistent hypertension in relation to histological findings immediately after delivery. No correlation is confirmable.

tion of glomerular capillary basement membrane in association with hypertension. The connection was not indicated by the results of the present study. 


\section{References}

1) Allen, A.C. The kidney, medical and surgical diseases, Grune and Stratton, New York, 1951.

2) Baird, D. \& Dann, J.S. J. Path. Bact., 1933, 37, 291.

3) Bell, E.T. Amer. J. Path., 1932, 8, 1.

4) Canny, A.J. Med. J. Austral., 1946, 2, 521.

5) Fahr, Th. Zbl.f. Jynäk., 1928, 52, 474.

6) Farquhar, M.G. Proceedings of 10 th annual conference on the nephrotic ryndrome, National Kidney Disease Foundation, Inc., New York, 1959.

7) Govan, A.J.T. J. Path. Bact., 1954, 67, 311.

8) Ishikawa, E. Suzuki, T., Sekiyama, S., Tanaka, H., Aizawa, S. \& Komori, R. Jap. J. Nephrol. 1960, 2, 301.

9) Kark, R.M., Pirani, C.L., Pollak, V.E., Muehrcke, R.C. \& Blainey, J.C. Ann. Int. Med., 1958, 49, 751 .

10) Leyden, E. Ztschr. f. klin. Med., 1886, 11, 26.

11) Löhlein, M. Dtsch. med. Wschr. 1918, 44, 1187.

12) McManus, J.F.A. Medical diseases of the kidney, Lea and Febriger, Philadelphia, 1950 .

13) Rinehart, J.F., Farquhar, M.G., Jung, H.C. \& Abul-Haj, S.K. Amer. J. Path., $1952,29,21$.

14) Sharma, G.P. J. Indian Med. Ass., 1956, 26, 445.

15) Spargo, B. McCartney, C.P. \& Winemiller, R. Arch. Path. 1959, 68, 593.

16) Way, G.T.C. \& Durham, N.C. Amer. J. Obstet. Gynec., 1947, 54, 928. 


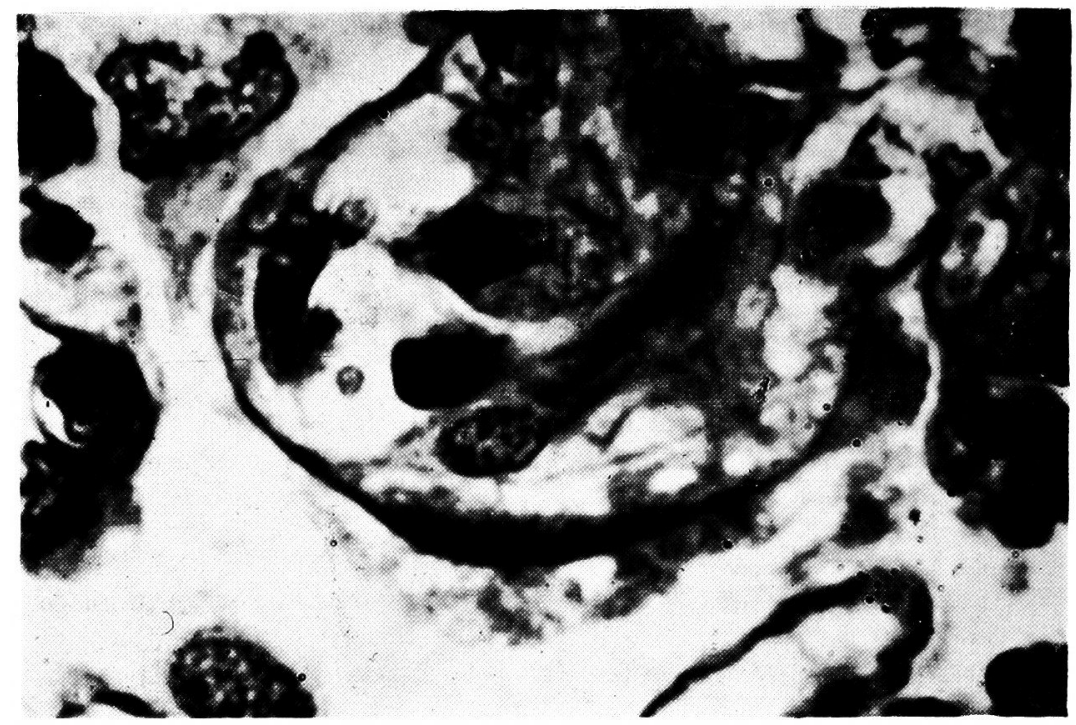

Fig. 5. Fibrinoid precipitate just inside capillary basement membrane at the lower side of the capillary loop in the middle of the figure. Swollen and vacuolated endothelial cell over the precipitate. Mesangial tissue is also swollen and edematous but without cellular proliferation. Initial glomerular lesion in toxemia of pregnancy.

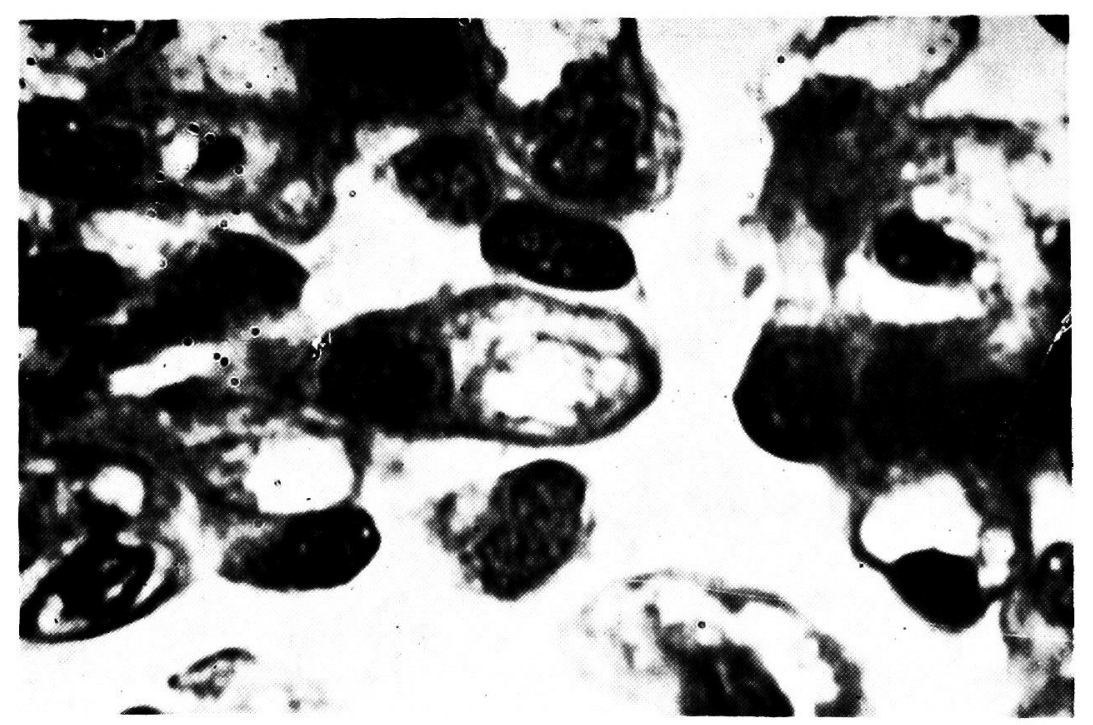

Fig. 6. The so-called "splitting" of capillary basement membrane in toxemia of pregnancy. Note apparent reduplication of basement membrane-like structure. This finding is histologically interpreted as edema of capillary basement membrane, but electron microscopical examinations usually reveal invasion of mesangial cells. 


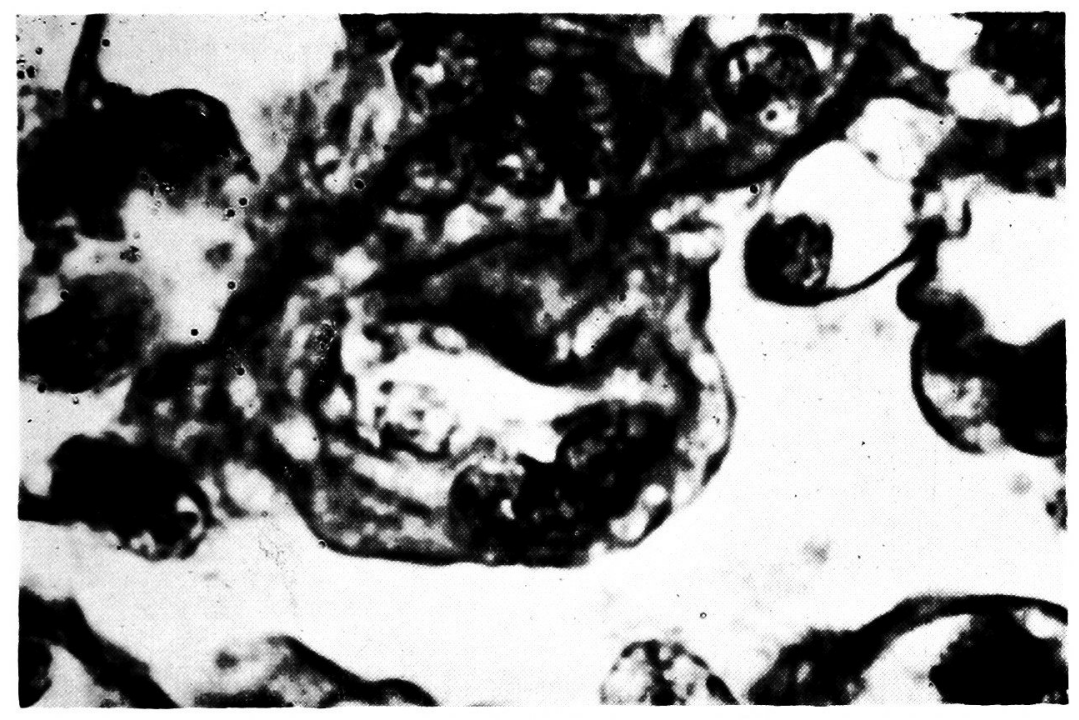

Fig. 7. Relatively advanced glomerular lesions in toxemia of pregnancy. Besides multiple layers of basement membrane-like structures nuclei are observed at the convexity of swollen "basement membrane". They are evidently of mesangial origin producing membranoous structures in the course of their invasion into lamina rara interna. 


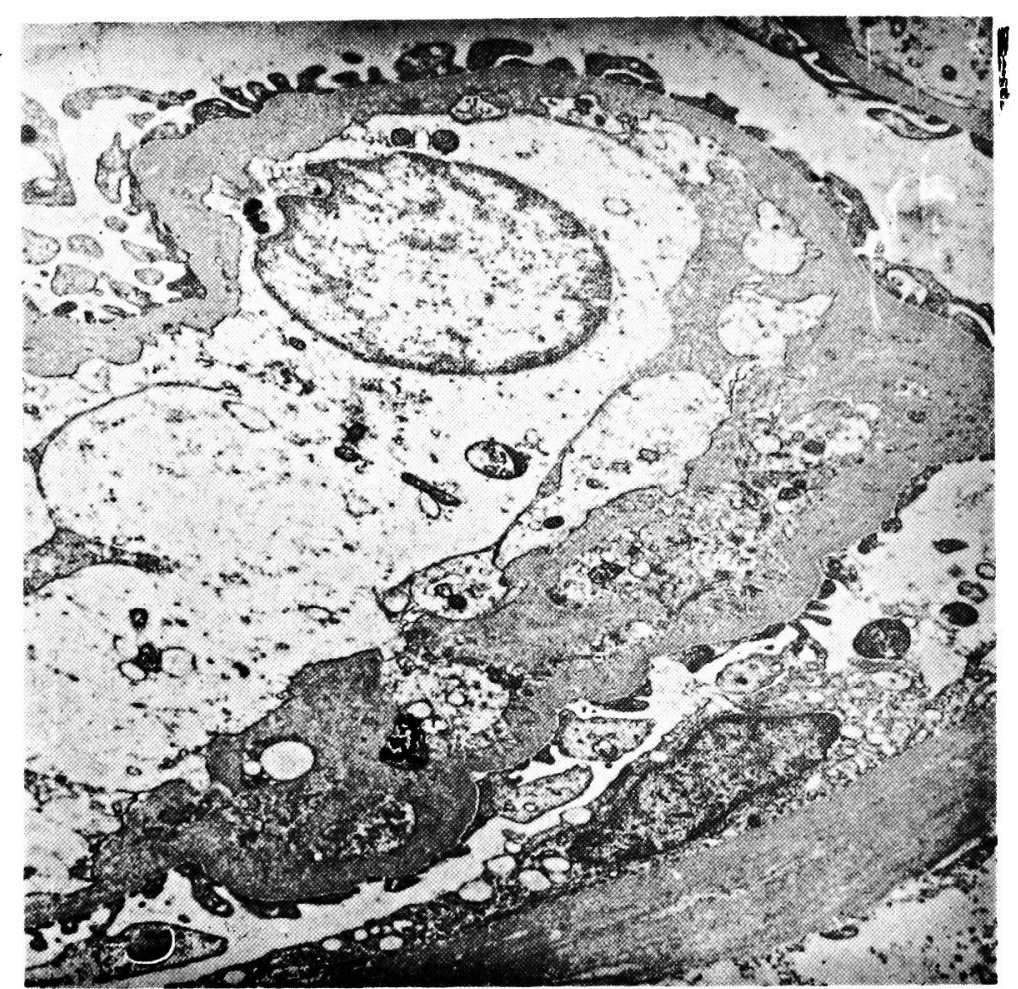

Fig. 8. Bulky and almost amorphous engorgement of endothelial cytoplasm demonstrated in electron microgram. 


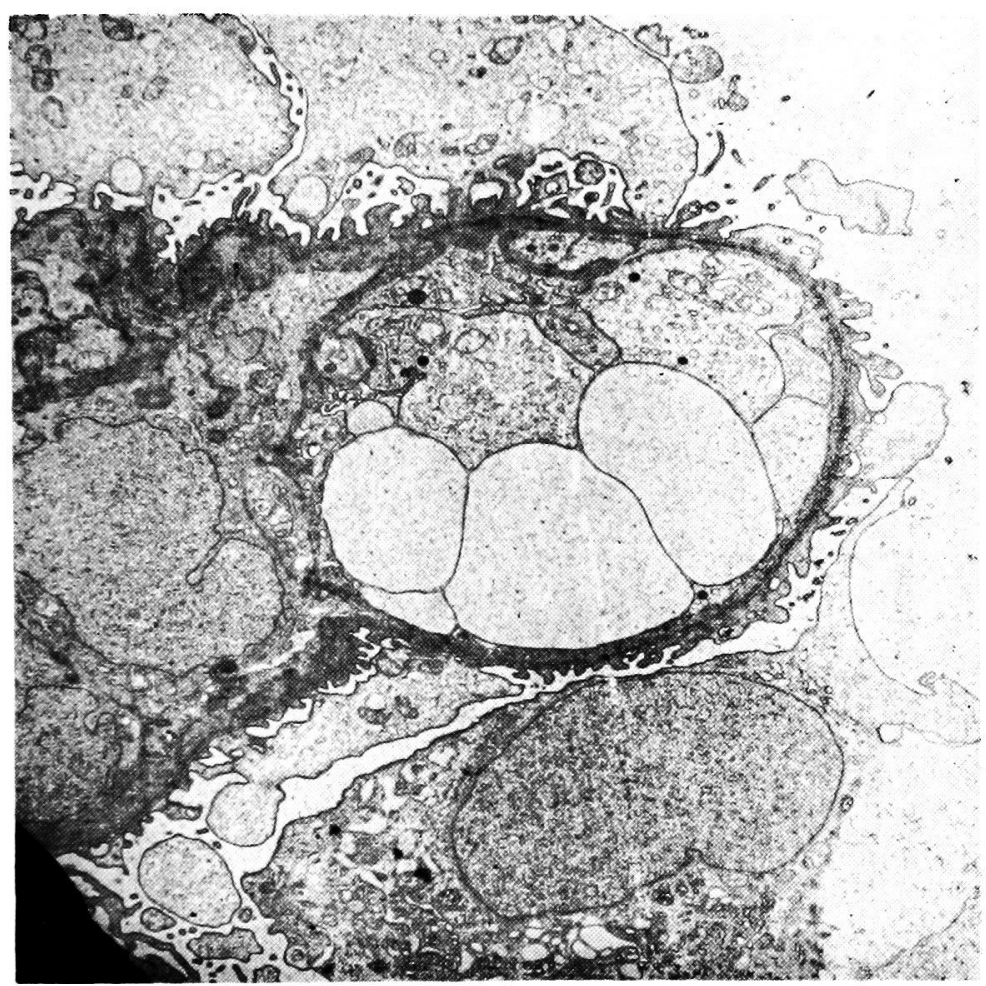

Fig. 9. Swollen and vacuolated endothelial cell completely obliterating capillary lumen. 


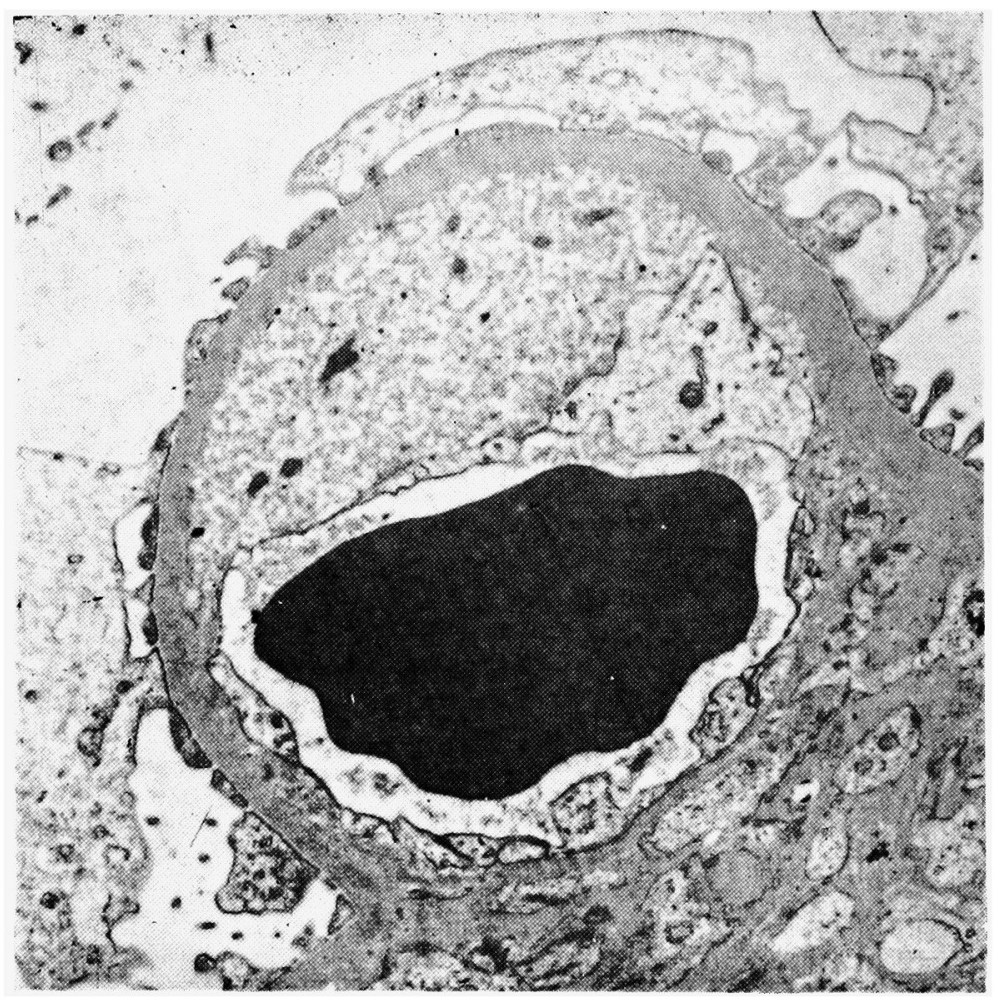

Fig. 10. Precipitation of granular substance probably of plasmal origin in lamina rara interna. Cytoplasm of an endothelial cell is demonstrated at the right side of the precipitate with attenuated cytoplasmic extension over the precipitate. 


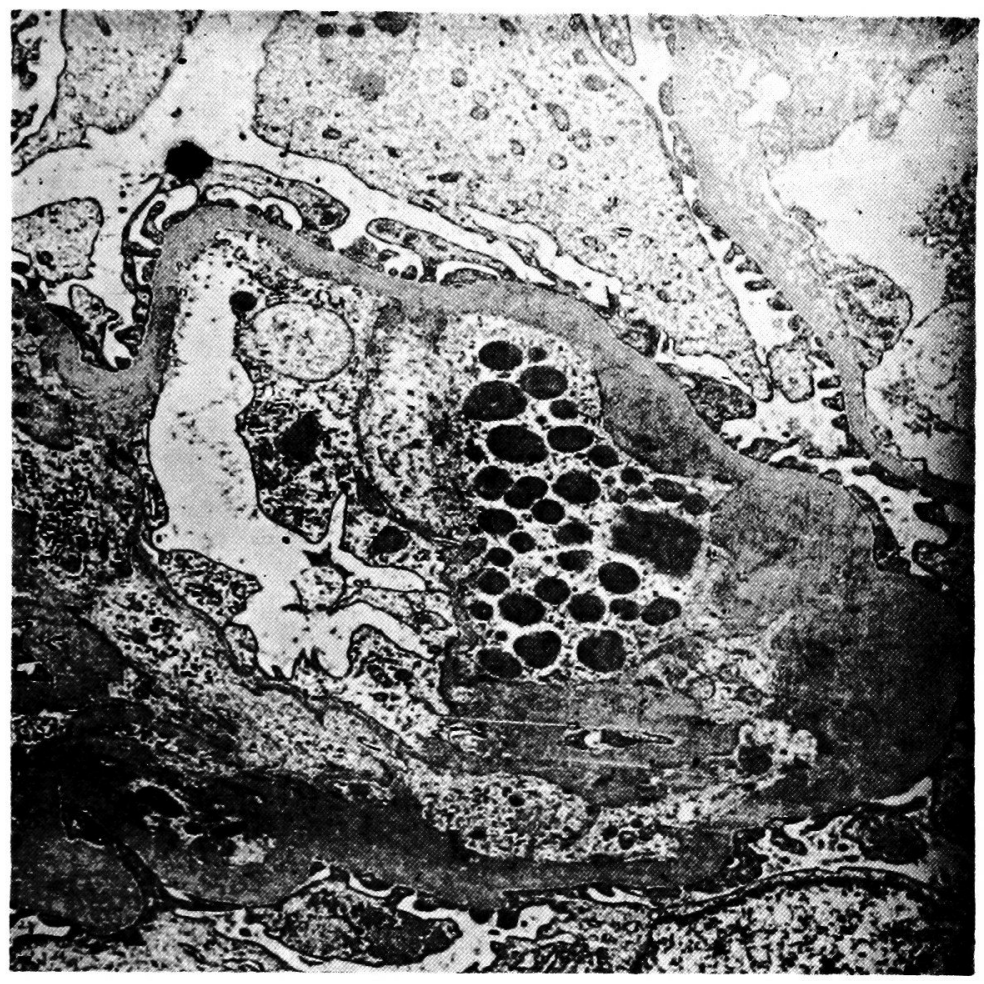

Fig. 11. Precipitation of coarse granular substances in lamina rara interna accompanied by invasion of mesangial elements with membraneous structures. 


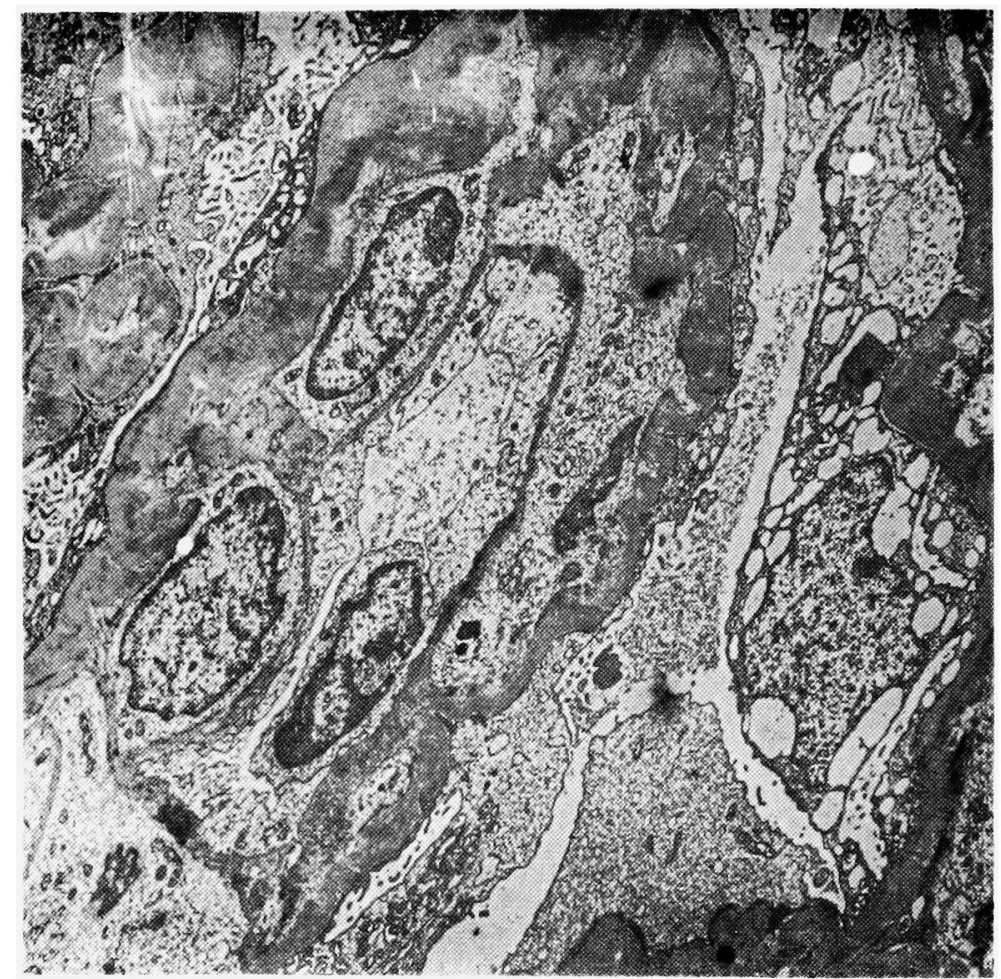

Fig. 12. Precipitation of granular substances of different appearance in lamina rara interna. 


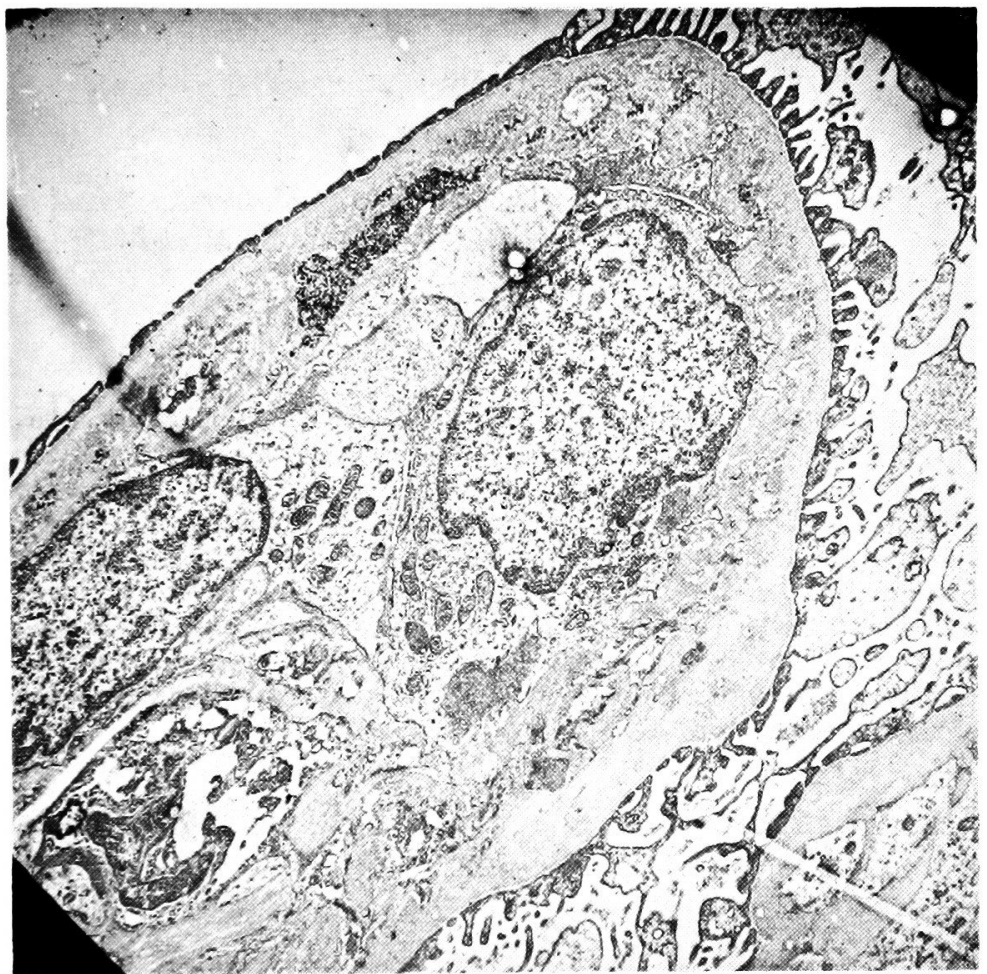

Fig. 13. Invasion of mesangial tissue into lamina rara interna producing membraneous structures and causing irregular undulation of pre-existing basement membrane. Epithelial foot processes are flattened and coalesced. 